\title{
Detecting Pulsing Denial-of-Service Attacks with Nondeterministic Attack Intervals
}

\author{
Xiapu Luo, Edmond W. W. Chan, and Rocky K. C. Chang \\ Department of Computing, The Hong Kong Polytechnic University, Hung Hom, Kowloon, SAR, Hong Kong \\ Correspondence should be addressed to Rocky K. C. Chang, csrchang@comp.polyu.edu.hk \\ Received 14 April 2008; Revised 29 October 2008; Accepted 21 January 2009 \\ Recommended by Chin-Tser Huang
}

\begin{abstract}
This paper addresses the important problem of detecting pulsing denial of service (PDoS) attacks which send a sequence of attack pulses to reduce TCP throughput. Unlike previous works which focused on a restricted form of attacks, we consider a very broad class of attacks. In particular, our attack model admits any attack interval between two adjacent pulses, whether deterministic or not. It also includes the traditional flooding-based attacks as a limiting case (i.e., zero attack interval). Our main contribution is Vanguard, a new anomaly-based detection scheme for this class of PDoS attacks. The Vanguard detection is based on three traffic anomalies induced by the attacks, and it detects them using a CUSUM algorithm. We have prototyped Vanguard and evaluated it on a testbed. The experiment results show that Vanguard is more effective than the previous methods that are based on other traffic anomalies (after a transformation using wavelet transform, Fourier transform, and autocorrelation) and detection algorithms (e.g., dynamic time warping).
\end{abstract}

Copyright (C) 2009 Xiapu Luo et al. This is an open access article distributed under the Creative Commons Attribution License, which permits unrestricted use, distribution, and reproduction in any medium, provided the original work is properly cited.

\section{Introduction}

Traditional denial-of-service (DoS) attacks are floodingbased DoS (FDDoS), which overwhelm a victim with a constant rate of useless packets. Moreover, several low-rate DoS attacks have recently emerged. These new attacks are able to attack TCP flows even more effectively than the FDDoS attacks in that their average attack rate could be much smaller for a similar damage. These attacks usually send a sequence of attack pulses to a victim router, and the TCP flows traversing it will periodically experience packet losses, thus seeing significant throughput degradation. The shrew attack [1], for example, confines a TCP sender to the timeout state by dispatching attack pulses at carefully chosen time instants. The reduction of quality (RoQ) attack [2] sends periodic attack pulses to force the victim router's active queue management mechanism to enter transient state. The pulsing denial-of-service (PDoS) attack [3] uses the attack pulses to cause victim TCP senders' congestion windows to drop frequently.

The low-rate attacks are harder to detect than the FDDoS attacks because of their low average attack rate and various attack patterns. Existing detection schemes are based on individual flows or aggregate flows. The methods in the flow-based detection scheme label a flow as malicious if it will periodically occupy a large portion of the bandwidth or cause packet loss in well-behaved flows, for example, [4-6]. However, this scheme is resource intensive, and characterizing a legitimate flow profile for various TCPbased applications is also very difficult. The aggregate-based detection scheme, on the other hand, detects attacks based on aggregated traffic statistics.

However, there are two major shortcomings to the aggregate-based detection mechanisms. First, all of them have been designed and tested only for a specific lowrate DoS attack. Therefore, they may not be effective for detecting other kinds of low-rate attacks and even the traditional FDDoS attack. For example, the two-stage detection algorithm proposed in [3] could not effectively detect the FDDoS attacks. Note that employing multiple detection algorithms is problematic and difficult to manage. Second, they have assumed specific attack scenarios, such as a constant attack period examined in [1-3]. An attack, however, can be easily launched under a different set of parameters (e.g., random intervals), which could render the detection algorithms ineffective. The anomalies in the power 
spectrum density, for example, may not exist if the attack period is not constant. The dynamic time warping approach becomes ineffective if the attack pulse's duration is longer than the sampling period.

In this paper we propose a single detection scheme, named, Vanguard, for the low-rate DoS attacks as well as the FDDoS attacks. Moreover, we do not assume a constant attack period for the low-rate DoS attacks. We will model the attacks as a sequence of attack pulses with arbitrary intensity and attack interval. This model therefore encompasses the shrew attack, RoQ attack, and PDoS attack. From this point on, we will refer to them collectively as polymorphic PDoS (PMDoS) attacks_-DoS attacks exist in many forms. In the Vanguard design, we first identify three traffic anomalies which are induced by the PMDoS attacks and then employ a change-point algorithm to detect them. To evaluate Vanguard's effectiveness, we have implemented it as a Snort plug-in [7]. Extensive testbed experiment results support that Vanguard is more effective and accurate than the previous approaches.

The rest of this paper is organized as follows. Section 2 discusses the previous detection algorithms proposed for low-rate DoS attacks. Section 3 presents the model for the PMDoS attacks considered in this paper. Section 4 presents the design of Vanguard. Section 5 presents the test-bed evaluation results to compare Vanguard with other detection methods. Section 6 finally concludes this paper with future work.

\section{Related Work}

Luo and Chang have proposed a two-stage detection system to detect PDoS attacks on the receiver side [3]. The detection is based on the presence of two traffic anomalies induced by the attacks: periodic fluctuations in the incoming TCP data traffic and a decline in the trend of the outgoing TCP acknowledgement (ACK) traffic. In the first stage, the system monitors the incoming data and outgoing ACK traffic using discrete wavelet transform. In the second stage, it employs a nonparametric CUSUM algorithm to detect the anomalies. We therefore refer to this system as WCM (wavelet and CUSUM). The experiment results show that the system is very effective in detecting the PDoS attacks with constant attack intervals. However, it will not be able to detect the FDDoS attacks with the same effectiveness because the attack will not cause periodic fluctuations in the TCP data traffic.

Another approach is based on a spectral analysis of the network traffic, and we refer to it as spectrum-based method (STM). Hussain et al. applied an STM method to differentiate between single-source and multisource DoS attacks [8]. Chen et al. have proposed a spectral template matching method to detect shrew attacks [9, 10]. They have observed that the power spectrum density of a traffic stream containing shrew attacks has much higher energy in low-frequency band as compared with legitimate traffic. Based on this observation, they have developed a scheme for collaborative anomaly detection. However, the STM approach will not be effective for general low-rate DoS attacks which could be easily tuned with different attack frequencies and intervals to evade the detection.

Sun et al. have proposed using dynamic time warping (DTW) to detect shrew attacks [11]. Similar to other approaches, there are two main stages. In the first stage, they use autocorrelation to extract the periodic patterns in the incoming network traffic. The autocorrelation is also used to eliminate the problem of time shifting. In the second stage, they use a slightly modified DTW algorithm to detect the signature of a shrew attack based on its autocorrelation. They have shown the differences between legitimate and attack traffic in their probability density functions of DTW. However, the DTW approach will not perform well if the attack pulses are not separated by a constant interval. Moreover, the DTW method will not be able to detect the FDDoS attacks effectively because the square-wave patterns, which are assumed by their method, do not exhibit in the traffic under attack.

D-WARD uses a useful metric that computes the ratio of the incoming TCP traffic to the outgoing TCP ACK traffic to detect DDoS attack [12]. Although Vanguard adopts the same metric, its use was different from D-WARD in two important aspects. First, D-WARD is placed in an attacker's source network and monitors traffic between the source network and a foreign host; Vanguard is located at the TCP receiver side and monitors all incoming and outgoing TCP traffic. Second, D-WARD uses a fixed ratio of 3 to distinguish an attack flow from legitimate ones; Vanguard employs a nonparametric CUSUM algorithm to identify abrupt changes in the ratio.

\section{The Polymorphic DoS Attacks}

We model a PMDoS attack as a sequence of attack pulses. Each attack pulse lasts for a short period of time $T_{\text {on }}>0$, and its intensity is given by $R_{a}$ bits per second (bps). Two adjacent pulses are separated by an interval $T_{\text {off }} \geq 0$. Generally, $T_{\text {on }}$, $T_{\text {off, }}$ and $R_{a}$ can assume any acceptable values. However, to facilitate the ensuing discussion, we consider a constant $R_{a}$. Note that the PMDoS attacks include the shrew, RoQ, PDoS, and FDDoS attacks as special cases. That is, the PMDoS attack is equivalent to a PDoS or RoQ attack when both $T_{\text {on }}$ and $T_{\text {off }}$ are constant. Moreover, if $T_{\text {off }}$ is close to 1 second and $T_{\text {on }}$ is approximately equal to the round-trip time (RTT) of the victim TCP flows, the PMDoS attack is equivalent to the shrew attack. Furthermore, when $T_{\text {off }}$ goes to 0 , the PMDoS attack becomes an FDDoS attack.

It is useful to consider two classes of PMDoS attacks separately. The first class is the FDDoS attacks when $T_{\text {off }}=0$. Let $R_{n}$ be the bandwidth of the victim router where packets in the victim TCP flows are dropped due to the attack. The FDDoS attack could be a low-rate attack (i.e., $R_{a}<R_{n}$ ) or a full-fledge attack (i.e., $R_{a}=R_{n}$ ). We refer to this class of attacks as flooding attacks. The second class is when $T_{\text {off }}>0$. In this case, it is possible that $R_{a}>R_{n}$, but the average attack rate $\bar{R}_{a}$ must be less than $R_{n}$. We refer to the second class of attacks as pulsing attacks [3]. Both attacks will cause packet losses to victim TCP flows. A less severe packet loss will 
cause the flows to enter the fast retransmit and fast recovery state, and a more severe one will induce timeout events. Both cases will effectively reduce the throughput in the victim TCP flows. We also define the attack cost by $\gamma=\bar{R}_{a} / R_{n}$.

In this paper we assume that the attacker sends pulses of useless TCP data packets in a PMDoS attack. The attacker therefore does not need to establish TCP connections to launch such attacks. Since the attack packets are also TCP, they will share the same queue as the legitimate TCP packets and will cause packet losses to these legitimate flows. Although the attack packets generally could have various adverse effects on routers, such as consumption of CPU and memory, we focus only on the effect of congesting the router buffers. Using ICMP and UDP packets for the attacks is also possible, but they may not disrupt legitimate TCP flows because routers will classify and buffer different types of traffic in separate queues. Moreover, we do not consider using nonTCP-friendly flows to launch the attack because there are already effective mechanisms to detect and punish such malicious flows [13].

Vanguard detects PMDoS attacks from the side of TCP receivers by analyzing the incoming TCP data traffic and outgoing ACK traffic. Therefore, Vanguard is designed to detect attacks for multiple hosts placed behind it. These hosts are running TCP application clients to receive data from external networks. It is also assumed that the data and ACK traffic in a TCP flow can be observed by Vanguard. For singly-homed networks, this assumption is obviously valid. For multihomed networks, additional mechanisms may be needed to mirror the data or ACK traffic to Vanguard for analysis. Furthermore, the incoming data traffic observed by Vanguard may not contain all the attack packets involved because many attack packets will be dropped at the bottleneck router. Moreover, these attack packets could carry different destination addresses or have low IP time-to-live values. Therefore, if a legitimate TCP flow is attacked at a router which is located before Vanguard on the forwarding path, many attack packets may not be observable to Vanguard. We will consider traffic anomalies for these two cases separately in the next section.

\section{Vanguard: A New Anomaly-Based Detection Scheme for the PMDoS Attacks}

In this section, we will first present three traffic anomalies caused by a PMDoS attack. After that, we introduce Vanguard, a new anomaly-based detection scheme for the PMDoS attacks.

\subsection{Three Traffic Anomalies Induced by the PMDoS Attacks}

4.1.1. Traffic Anomaly for Observable Attack Traffic. When the bulk of the attack traffic is present in the incoming data traffic, Vanguard uses an anomalous increase in the ratio of the incoming TCP traffic to the outgoing TCP ACK traffic to detect the PMDoS attacks. Normally this ratio, in terms of the number of data and ACK packets, will fall between one (due to duplicate ACK packets [14]) and two (due to the
ACK-every-other-data-segment strategy [14]). However, the PMDoS attack packets will inflate the ratio because the attack traffic will significantly increase the number of TCP data packets. On the other hand, the ACK traffic will decrease as a result of the drop in the legitimate TCP data.

4.1.2. Traffic Anomalies for Unobservable Attack Traffic. When the attack traffic is not significant in the incoming data traffic, Vanguard uses two other anomalies for the detection purpose. The first is an anomalous decline in the outgoing TCP ACK traffic. An obvious effect of a PMDoS attack is a decline in the outgoing TCP ACK traffic because the victim TCP flows drop their sending rates. This anomaly has also been used in [3] to detect PDoS attacks. However, this anomaly alone will cause many false alarms when the ACK traffic decline is due to a normal decrease in the data traffic. To decrease the false alarms, Vanguard utilizes a second anomaly: an anomalous change in the distribution of the incoming TCP data rate. Besides the ACK traffic decline, a PMDoS attack will also perturb the distribution of the victim flows' data traffic. For example, as shown in Figure 1(a), a pulsing attack will force the victim TCP senders' cwnd to converge to a low value. A flooding attack will also constrain the victim TCP flows' cwnd, as shown in Figure 1(b). However, the fluctuation of the cwnd for the flooding attack is modulated by the constrained bandwidth rather than the attack pulses.

4.2. Vanguard: A New Detection Scheme. Vanguard detects the PMDoS attacks based on the three traffic anomalies just described. Vanguard first constructs three corresponding statistics: $r_{d}$ for the TCP data rate in bps, $r_{a}$ for the TCP ACK rate in bps, and $\delta_{f}$ for the absolute change in the TCP data-rate distribution. If there is no change in the data-rate distribution, $\delta_{f}=0$; otherwise, $\delta_{f}>0$. We will discuss how they are measured shortly. Vanguard also computes $r_{d / a}=$ $r_{d} / r_{a}$, where $r_{d}$ and $r_{a}$ are measured in number of packets per second. Based on the two attack scenarios discussed in the last section, Vanguard will raise an alarm if the statement below is true:

$$
r_{d / a} \uparrow \vee\left\{r_{a} \downarrow \wedge \delta_{f} \uparrow\right\},
$$

where $\uparrow$ and $\downarrow$ represent abrupt increase and abrupt decrease, respectively. An abrupt change in the rates means a sharp (positive or negative) change in the rates, whereas an abrupt increase in $\delta_{f}$ means a significant change in the distribution. As we will see later, Vanguard employs a nonparametric change-point detection algorithm to detect the abrupt changes.

4.2.1. Measuring TCP Data Rate and ACK Rate. Vanguard makes a detection decision at the end of a detection window of $T_{w}$ seconds. For computing a sample data rate and a sample ACK rate, Vanguard first obtains $N_{w}$ observations for the volume of data and ACK packets in bytes uniformly over the detection window. Denote the respective values by $m_{d}(i)$ and $m_{a}(i)$ for the $i$ th observation. Vanguard then obtains the $n$th 

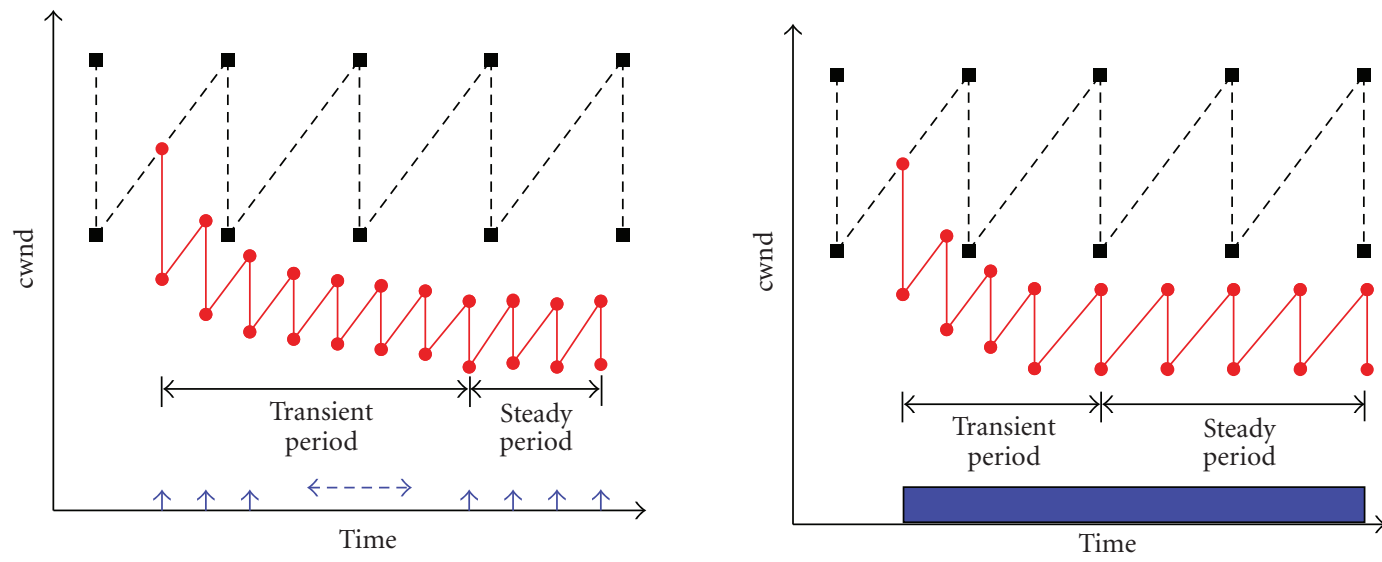

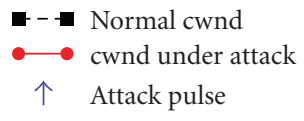

(a) Under a pulsing attack

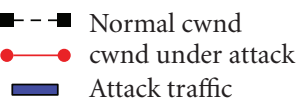

(b) Under a flooding attack

Figure 1: The evolution of cwnd under a PMDoS attack [3].

sample for the data rate and ACK rate, denoted by $r_{d}(n)$ and $r_{a}(n)$, by

$$
\begin{aligned}
& r_{a}(n)=\frac{1}{T_{w}} \sum_{i=(n-1) N_{w}+1}^{n N_{w}} m_{a}(i), \\
& r_{d}(n)=\frac{1}{T_{w}} \sum_{i=(n-1) N_{w}+1}^{n N_{w}} m_{d}(i) .
\end{aligned}
$$

Vanguard computes $r_{d / a}(n)=r_{d}(n) / r_{a}(n)$, where $r_{d}(n)$ and $r_{a}(n)$ are measured in number of packets per second.

\subsubsection{Measuring Changes in TCP Data-Rate Distribution.} Vanguard employs the color histogram indexing method [15] to capture the change in the distribution. In the field of image retrieval, it has been proven a robust method of computing the similarity of two images [16]. In a similar way, Vanguard uses it to measure the similarity between two TCP data-rate distributions: the ones with and without the PMDoS attacks. The similarity index for Vanguard is $\delta_{f}(n)$. An abrupt change in the sequence of $\delta_{f}(n)$ will raise an alarm for a possible onset of a PMDoS attack.

Vanguard computes $\delta_{f}(n)$ by first generating a histogram for the observations collected in the $n$th detection window. To do so, it constructs $B$ histogram bins for $m_{d}(i)$ obtained from the nth detection window. Each bin's width is given by $\left(m_{d}^{\max }-m_{d}^{\min }\right) / B$, where $m_{d}^{\max }$ and $m_{d}^{\min }$ are the maximum and minimum values of the observations. The traffic histogram is therefore given by $h(n)=\left(h_{n, 1}, \ldots, h_{n, B}\right)$, where $h_{n, k}$ is the fraction of the observations falling into the $k$ th bin. Vanguard then derives a cumulative histogram $(\mathrm{CH})$ $H(n)=\left(H_{n, 1}, \ldots, H_{n, B}\right)$ from $h(n): H_{n, i}=\sum_{k=1}^{i} h_{n, k}$.

For detecting an anomalous data-rate distribution based on the $\mathrm{CH}$, Vanguard is also provided with a $\mathrm{CH}$ for the data rates of attack-free TCP traffic which is denoted by
$\hat{H}=\left(\hat{H}_{1}, \ldots, \hat{H}_{B}\right)$. A set of training data is usually provided for deriving the $\mathrm{CH}$ and also other parameters for the detection algorithm in use (see the next section on changepoint detection). Vanguard uses the Euclidean distance for computing $\delta_{f}(n)$ :

$$
\delta_{f}(n)=\sqrt{\sum_{k=1}^{B}\left(H_{n, k}-\hat{H}_{k}\right)^{2}} .
$$

4.2.3. Change-Point Detection. Vanguard uses the CUSUM algorithm to detect abrupt changes in the sequences of $r_{a}(n)$, $r_{d / a}(n)$, and $\delta_{f}(n)$. The CUSUM algorithm has been successfully applied to tackle many signal processing problems [17]. The algorithm assumes that the mean of the variables being monitored will change from negative to positive. However, $r_{a}, r_{d / a}$, and $\delta_{f}$ are always nonnegative under an attack-free environment. Vanguard therefore transforms them into three new random sequences:

$$
\begin{aligned}
s_{a}(n) & =\alpha_{a}-r_{a}(n), \\
s_{d / a}(n) & =r_{d / a}(n)-\alpha_{d / a}, \\
s_{\delta}(n) & =\delta_{f}(n)-\alpha_{\delta},
\end{aligned}
$$

where $\alpha_{a}, \alpha_{d / a}$, and $\alpha_{\delta}$ are constants. Since a PMDoS attack will decrease $r_{a}(n)$ and increase $r_{d / a}(n)$ and $\delta_{f}(n)$, the attack will increase the values of $s$. $(n)$ 's. If the increases are significant enough, the $s .(n)$ 's will become positive, thus resulting in abrupt changes to the three monitored sequences.

To determine the values of $\alpha_{a}, \alpha_{d / a}$, and $\alpha_{\delta}$, a set of attack-free training data is needed. Vanguard computes from the training set the average and standard deviation for $r_{a}$ (denoted by $\operatorname{avg}\left(\hat{r}_{a}\right)$ and $\operatorname{std}\left(\hat{r}_{a}\right)$ ), the maximum value for 


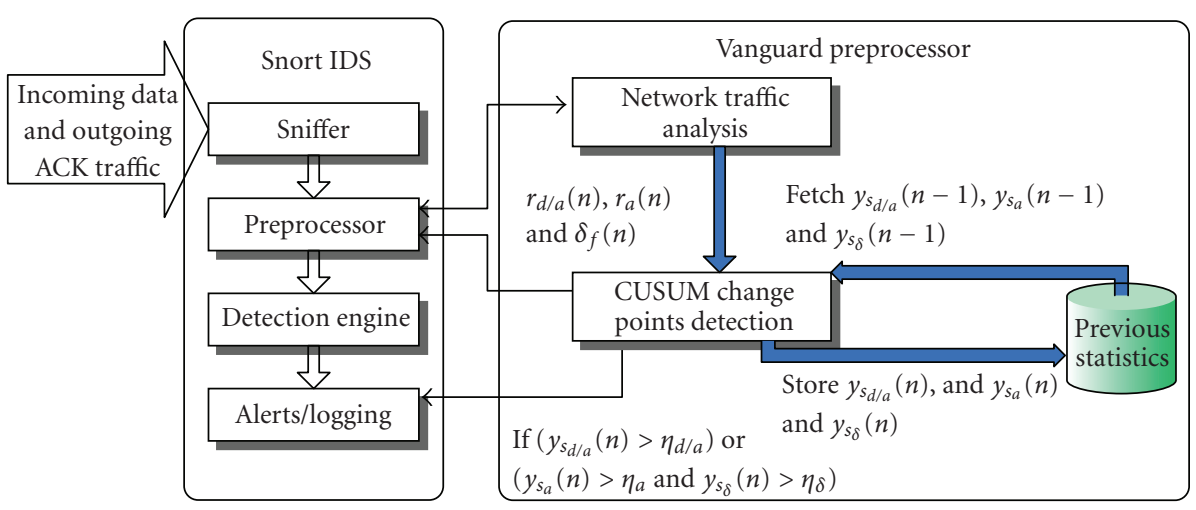

FIgURE 2: A Snort implementation of Vanguard.

$r_{d / a}\left(\right.$ denoted by $\left.\max \left(\hat{r}_{d / a}\right)\right)$, and the maximum value for $\delta_{f}$ (denoted by $\left.\max \left(\hat{\delta}_{f}\right)\right)$. Vanguard then sets

$$
\begin{aligned}
\alpha_{a} & =\operatorname{avg}\left(\hat{r}_{a}\right)-\beta \times \operatorname{std}\left(\hat{r}_{a}\right), \\
\alpha_{d / a} & =\max \left(\hat{r}_{d / a}\right), \\
\alpha_{\delta} & =\max \left(\hat{\delta}_{f}\right) .
\end{aligned}
$$

Note that we could have set $\alpha_{a}=\operatorname{avg}\left(\hat{r}_{a}\right)$. However, to provide flexibility in configuring Vanguard, we have introduced $\beta-a$ configurable parameter that determines Vanguard's sensitivity to the decline in the ACK rate. The value of $\beta$ is usually set to 1 or 2 .

We denote the CUSUM values of $s_{a}(n)$ by $y_{s_{a}}(n)$ which is obtained by

$$
\begin{gathered}
y_{s_{a}}(n)=\max \left\{0, y_{s_{a}}(n-1)+s_{a}(n)\right\}, \quad n \geq 1, \\
y_{s_{a}}(0)=0 .
\end{gathered}
$$

The presence of an anomalous decline in the outgoing ACK traffic is confirmed if $y_{s_{a}}(n)>\eta_{a}$, where $\eta_{a}$ is the corresponding CUSUM threshold. Similarly, by comparing the CUSUM values $y_{s_{d / a}}(n)$ and $y_{s_{\delta}}(n)$ with the corresponding CUSUM thresholds $\eta_{d / a}$ and $\eta_{\delta}$, Vanguard can confirm an anomalous increase in the ratio of data and ACK rates and an anomalous change in the data-rate distribution.

\section{Performance Evaluation}

To evaluate the performance of Vanguard, we have implemented Vanguard as a preprocessor plug-in in a Snort intrusion detection system (IDS) [7] and conducted experiments on a testbed. We have also compared the WCM, DTW, and STM methods discussed in Section 2 with Vanguard.

5.1. A Snort implementation of Vanguard. Figure 2 depicts the architecture of our Snort implementation of Vanguard. After the Vanguard preprocessor is registered in the Snort's preprocessor list through the function AddFuncToPreprocList(), Snort starts intercepting the incoming TCP data traffic and outgoing ACK traffic for the hosts under its protection and forwards them to the Network Traffic Analysis (NTA) unit in the Vanguard preprocessor.
The NTA unit records the packet size and updates the corresponding packet counter for the current sampling interval. Whenever $N_{w}$ continuous observations (a detection window) have been collected, they evaluate $r_{a}, r_{d / a}$, and $\delta_{f}$ according to (2) and (3) and sends them to the CUSUM Change-Points Detection (CCPD) unit. The CCPD unit is responsible for detecting PMDoS attacks using the CUSUM algorithm and the detection rule in (1). If an alarm is raised, it will immediately call the function SnortEventqAdd() to pass a PMDoS attack alert to the Snort's Alert/Logging module. Note that our Vanguard implementation does not use Snort's detection engine.

Before the Vanguard preprocessor begins the PMDoS attack detection process, the preprocessor has to first determine the constant values $\left(\alpha_{a}, \alpha_{d / a}, \alpha_{\delta}, \eta_{a}, \eta_{d / a}\right.$, and $\left.\eta_{\delta}\right)$ using a set of training data. The preprocessor therefore provides a facility to specify the length of the training period, in terms of the number of continuous detection windows (denoted by $N_{d}$ ), before using it for detection. At the end of the training period, it computes $\alpha_{a}, \alpha_{d / a}$, and $\alpha_{\delta}$ according to (5), respectively, and sets the CUSUM thresholds $\eta_{a}$, $\eta_{d / a}$, and $\eta_{\delta}$ to the means of the sequences $\left\{\left|s_{a}(n)\right|\right\}_{n=1}^{N_{d}}$, $\max \left\{\left\{\left|s_{d / a}(n)\right|\right\}_{n=1}^{N_{d}}, 2.5\right\}$, and $\left\{\left|s_{\delta}(n)\right|\right\}_{n=1}^{N_{d}}$, respectively. To reduce the number of false alarms in the Vanguard detection, we have applied a minimum threshold (i.e., 2.5) for $\eta_{d / a}$. However, we do not apply it to $\eta_{a}$ and $\eta_{\delta}$ because normal TCP data and ACK traffic rates could vary significantly.

5.2. A Testbed. Figure 3 shows a general testbed for evaluating Vanguard and other detection schemes. The testbed consists of $b+1$ routers. All the links, except for the bottleneck link (the last link) between $X_{b}$ (the bottleneck router) and $X_{b+1}$, have a one-way propagation delay of $T_{x}$ milliseconds and a capacity of $R_{x}$ Mbps. The bottleneck link, on the other hand, has a one-way propagation delay of $T_{b}$ milliseconds and a capacity of $R_{b} \mathrm{Mbps}$, and does not carry cross-traffic. The $N_{s}$ long-lived legitimate TCP flows traverse all routers and arrive at the receivers. Moreover, there are $N_{c}$ crosstraffic sources of long-lived TCP flows competing for the router resources. A PMDoS attacker generates attack traffic destined to the receivers. Therefore, the legitimate end-toend TCP flows will suffer from packet losses at $X_{b}$. Vanguard 


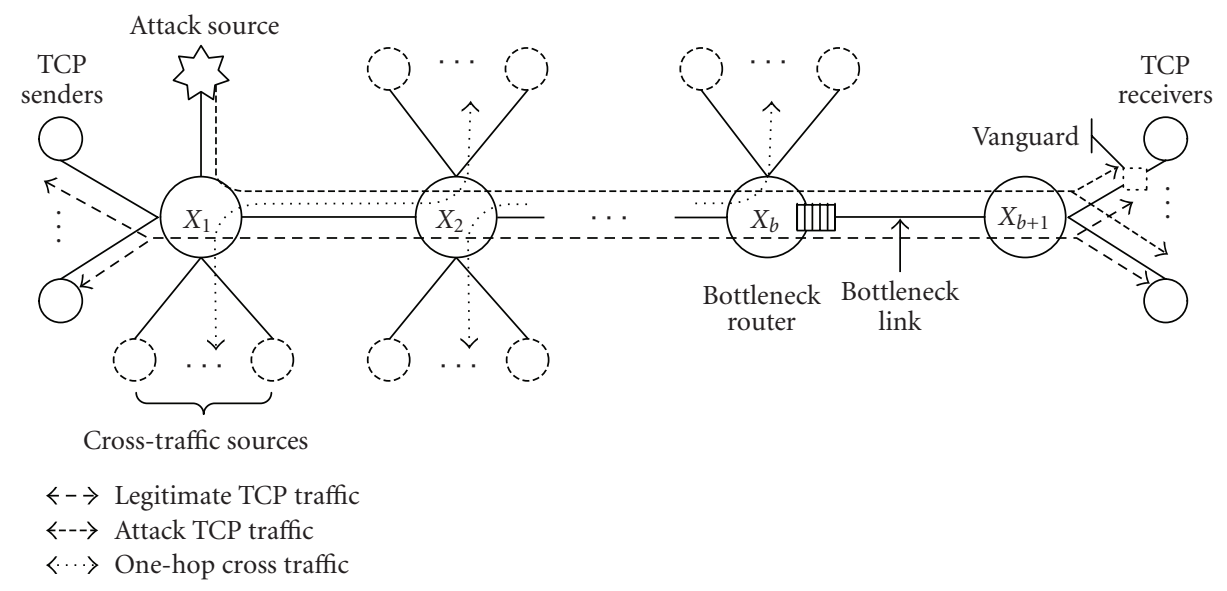

Figure 3: A general testbed for the empirical evaluation of Vanguard and other detection schemes.

performs detection based on the traffic observed from a receiver's link connected to $X_{b+1}$.

In our testbed evaluation to be presented next, we have used the following settings: $b=2$ (three routers), $N_{s}=15$ (TCP New Reno), $N_{c}=10$ (TCP New Reno), $T_{x}=15$ milliseconds, $T_{b}=30$ milliseconds, $R_{x}=100 \mathrm{Mbps}$, and $R_{b}=10 \mathrm{Mbps}$. Each legitimate TCP flow experiences a fixed RTT of 150 milliseconds (denoted by $r t t$ ) and employs a minimum retransmission timeout value of $1 \mathrm{~s}$. The three routers' hardware configurations are Pentium III/500 Mhz with $256 \mathrm{MB}$ RAM running FreeBSD v4.9. The bottleneck router $X_{b}$ is configured with Dummynet [18] to simulate a Random Early Detection (RED) [19] queue of size $Q=$ $\left(r t t \times R_{b}\right) / 8$ bytes. We have adopted the RED parameters suggested in [20]: $\max _{\mathrm{th}}=0.7 \mathrm{Q}, \min _{\mathrm{th}}=0.2 \mathrm{Q}, w_{q}=0.002$, and $\max _{p}=0.1$. We have also set-up another RED queue in $X_{b}$ with the same parameter settings for the outgoing TCP ACK traffic. The hardware configurations of all TCP senders/receivers are Pentium 4/1.5 GHz with 512 MB RAM running Linux kernel v2.6.5. The attacker has the same hardware configurations and is running Windows XP SP1.

For the PMDoS attacks, we have considered nine attack costs: $\gamma=0.1,0.2, \ldots, 0.9$. In addition, we have tried out six different attack configurations to achieve a given attack cost: $T_{\text {on }}=\{150,200,250\}$ milliseconds and $R_{a}=\{20,40\}$ Mbps. Although the attack cost is the same, these six configurations are expected to have different impacts on the legitimate flows. An attack with higher $T_{\text {on }}$ and $R_{a}$ will cause more packet losses in a single attack pulse. We have set the minimum $T_{\text {on }}$ to $r t$ (i.e., $150 \mathrm{~ms}$ ) in order to maximize the impact of an attack pulse on the victim TCP flows. Choosing a $T_{\text {on }}<r t t$, on the other hand, will have less impact because the attack pulse could miss many TCP flows. We have applied these 54 scenarios to both pulsing and periodic attacks. We have also experimented with the FDDoS attacks using the nine attack costs. As a result, we have evaluated Vanguard and other detection systems based on a total of $117(54 \times 2+9)$ different attack scenarios.

The experiment for each scenario lasts for 370 seconds. At the 131st seconds, the attacker launches a PMDoS attack that lasts to the end of the experiment. We have implemented the PMDoS attack traffic generator using WinPcap v3.0 [21]. Both the legitimate flows and cross traffic are generated using Iperf v1.7.0 [22]. We have employed the Snort implementation of Vanguard with the following settings: $T_{w}=5$ seconds and $N_{w}=1000$ to achieve a small detection delay, and $N_{d}=20$ (a training period of 100 seconds) to obtain an adequate training period. Moreover, Vanguard uses $B=25$ for computing $\delta_{f}(n)$ and $\beta=2$ for computing $\alpha_{a}$. The detection time of 240 seconds (i.e., 370-130 seconds) therefore corresponds to an unsuccessful detection.

5.3. A Testbed Evaluation of Vanguard. Figures 4, 5, and 6 illustrate the Vanguard detection of a periodic pulsing attack (i.e., the attack interval is a nonzero constant), a stochastic pulsing attack (i.e., the attack interval is random), and a flooding attack (i.e., the attack interval is 0 ), respectively. The data are based on $\gamma=0.6$ for both flooding and pulsing attacks. The periodic and stochastic pulsing attacks use $R_{a}=$ $30 \mathrm{Mbps}$ and $T_{\text {on }}=150$ milliseconds.

Subfigure (a) shows the raw incoming TCP traffic in the upper panel and the raw outgoing ACK traffic in the lower panel. Subfigures (b)-(d) plot the respective sequences of $r_{a}(n), r_{d / a}(n)$, and $\delta_{f}(n)$. In each of them, the upper panel shows the raw data of the statistics, and the lower panel shows the CUSUM detection results of these statistics. We can observe from subfigure (a) that the data and ACK traffic exhibit abrupt changes at the onset of the attack (i.e., at the 131st seconds). There is a similar drop in the ACK rate across the three attack scenarios. However, the impacts on the data rates are not entirely the same. In particular, the variability in the data rate for the flooding attack is much less than the other two. The subfigures (b)-(d) also show that the CUSUM can effectively detect the onsets of the three attacks.

Figure 7 plots the total time required for detecting the PMDoS attacks against the attack cost for the 117 attack scenarios. Each symbol represents the detection time for a scenario. Note that the results for flooding attacks are present in both subfigures. Figure 7(a) shows the results for the periodic pulsing attacks, and Figure 7 (b) shows the 


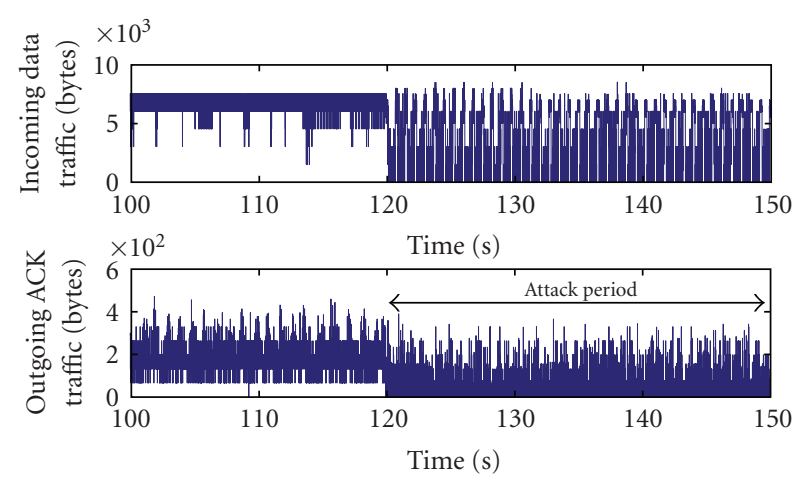

(a) A periodic pulsing attack
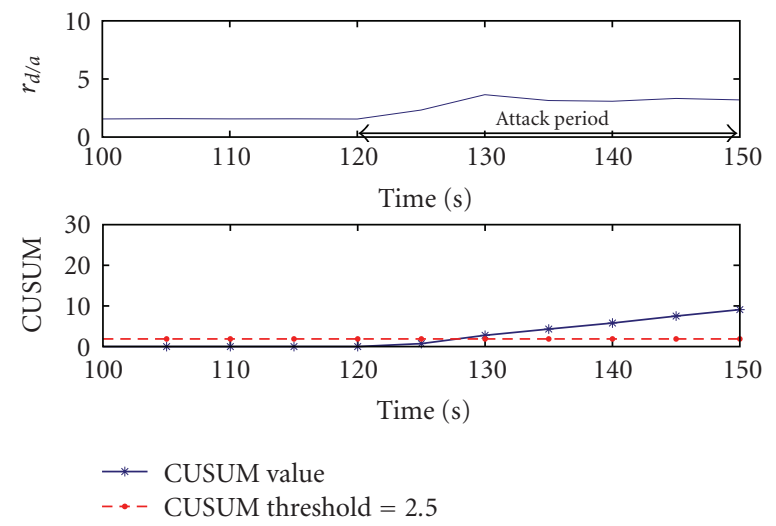

(c) $r_{d / a}$
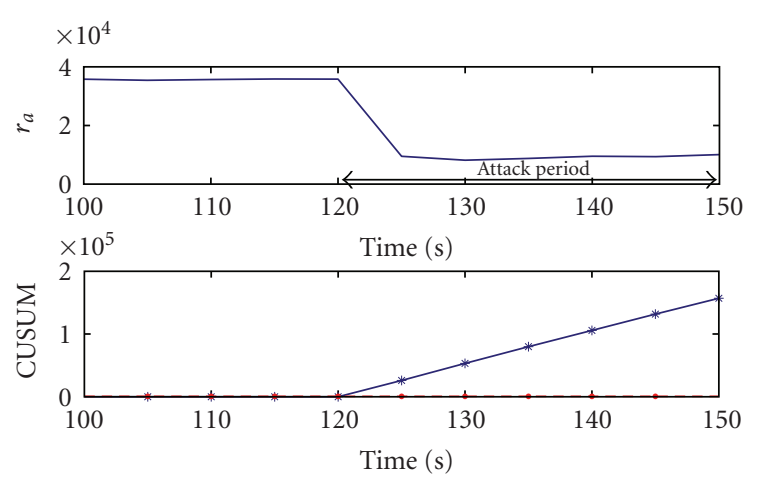

* CUSUM value

- - CUSUM threshold $=623.1$

(b) $r_{a}$
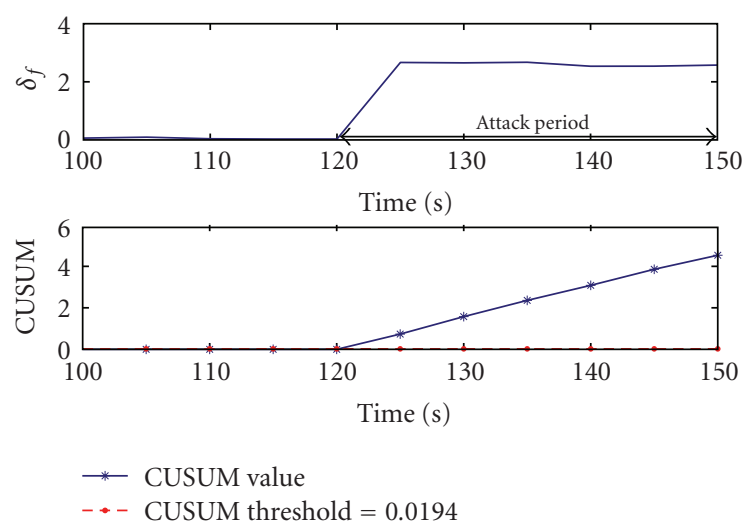

(d) $\delta_{f}$

FIgURE 4: Detecting periodic pulsing attacks using Vanguard.

results for the stochastic pulsing attacks. Each subfigure also includes the detection times for the flooding attacks. Note that Vanguard can identify all the attack scenarios within six detection windows (i.e., 30 seconds). In fact, it can detect all the flooding attacks immediately after the first detection window. It is not difficult to see why more time is required to confirm a less aggressive pulsing attack (i.e., with a small attack cost), particularly with stochastic attack intervals. We have also repeated the experiments using a Droptail queue with the same queue length as the RED queue. The experiment results show that Vanguard can also identify all the PMDoS attacks.

There are clearly tradeoffs in selecting between large and small detection windows. A small $T_{w}$ can speed up the Vanguard detection, but it is more sensitive to the surge of the monitored traffic. A too large $T_{w}$, on the other hand, will be too slow to detect an attack. Based on the experiment results, a suitable choice for our experiments is $T_{w}=5$ seconds. Another important Vanguard parameter is $B$ that determines the granularity of the traffic histogram. Our experiment results show that 25 bins gives good results for all experiments. The effect of noise could be significant when the bin size becomes larger. In such a finely quantized histogram, many bins will have a zero count (no traffic); therefore, a slight change in the traffic can result in a significant change in the resultant histogram, thus producing a false alarm.

5.4. Vanguard's False Positive Rates. To evaluate Vanguard's false positive rate (FPR), we turn to the real data traces because they contain realistic traffic dynamic which may not appear in our testbed environment. We have used TCP flows collected from 13 sets of the LBNL enterprise data traces [23] from October 2004 to January 2005 and nine sets of WIDE backbone data traces [24] from September 2005 to March 2006. To acquire an adequate training period, we have run Vanguard detection for the TCP flows containing at least 100 TCP data segments in either direction. We have set the training period to $44 \%$ of the longest lifetime of the target flows, so that the training periods for all the flows are not less than 20 seconds. Accordingly, we have obtained 62 and 49 TCP flows from the LBNL and WIDE trace sets for the evaluation, respectively. Other configuration settings for Vanguard remain unchanged.

Vanguard raised alert for one flow in both the LBNL trace set and WIDE trace set, thus yielding respective FPRs of $1.62 \%$ and $2.04 \%$. Moreover, both false alerts were due to the criterion of $r_{a} \downarrow \wedge \delta_{f} \uparrow$. The Vanguard's false alarms 


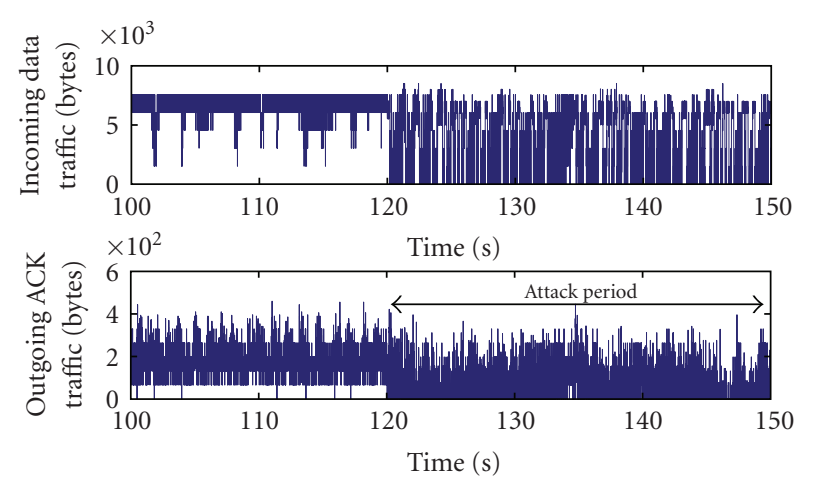

(a) A stochastic pulsing attack
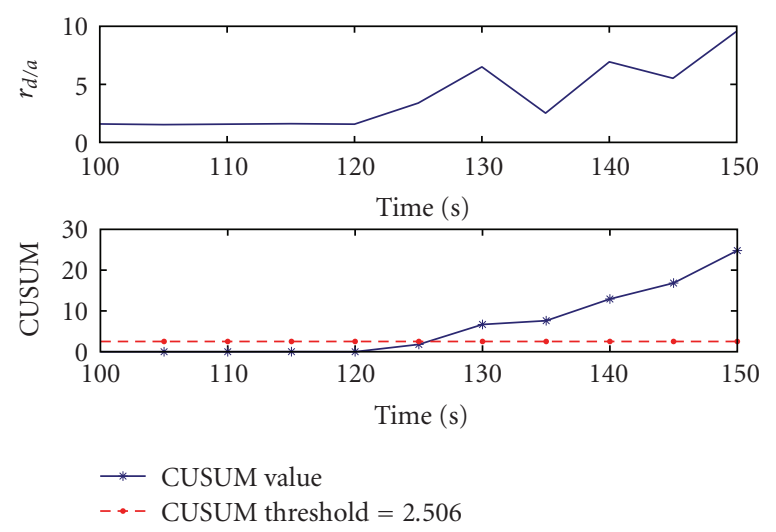

(c) $r_{d / a}$
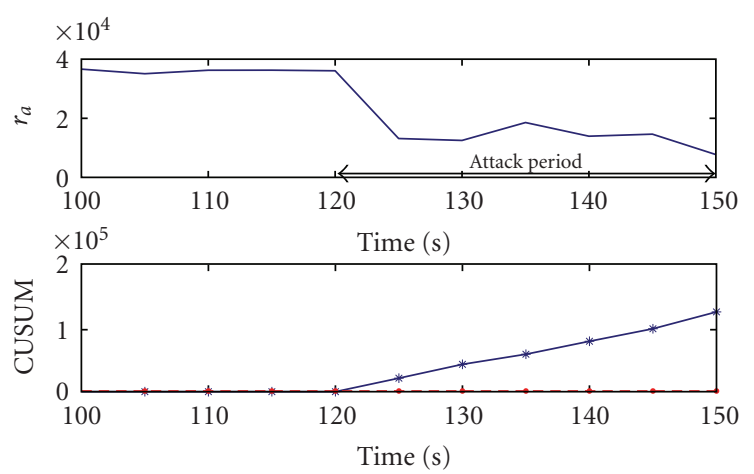

* CUSUM value

- CUSUM threshold $=1039$

(b) $r_{a}$
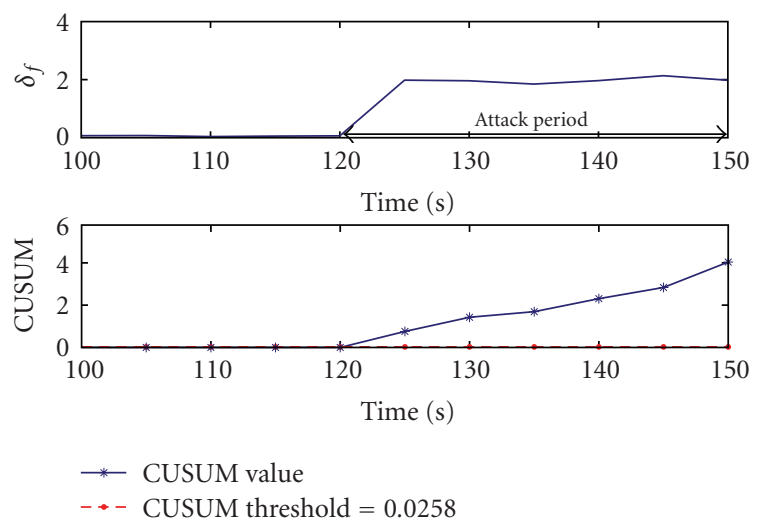

(d) $\delta_{f}$

Figure 5: Detecting stochastic pulsing attacks using Vanguard.

are due to the idle periods existing in both TCP data traffic and TCP ACK traffic. There are two consequences for the legitimate idle periods existing in the flow. First, these idle periods remain in the whole training period and thus result in "false" thresholds for $r_{a}$ and $\delta_{f}$. Therefore, a sudden increase in the TCP data traffic or TCP ACK traffic will make the detection rule in (1) true. However, the threshold for $r_{d / a}$ is not affected by the idle period because of the minimum threshold value of 2.5. Second, these idle periods abruptly decrease $r_{a}$ and increase $\delta_{f}$ during the Vanguard detection, and, as a result, the detection rule in (1) becomes true. A possible way to resolve this problem is to detect and skip these idle periods during the Vanguard detection. The idle periods could be identified by comparing the interpacket interval with a threshold.

5.5. Comparing with Other Detection Methods. We have also evaluated the WCM, DTW, and STM methods and compared their performance with Vanguard. We have implemented the WCM [3], DTW [11], and STM [8] methods in MATLAB and obtained their performance using the data traces captured from the testbed experiments conducted for Vanguard. Therefore, the legitimate and attack traffic used for the comparisons are the same as for Vanguard's evaluation.
5.5.1. The WCM Method. Figure 8 shows the average detection time versus the attack cost for the WCM method. For the WCM method's configurations, we have set each sampling window to 12.8 seconds to achieve a small detection delay and $N_{d}=6$ to obtain a training period of 76.8 seconds. The remaining configurations are the same as those used in [3]. The average detection rate is $92.31 \%$. Although the WCM method can discover all the ongoing periodic and stochastic pulsing attacks within three detection windows (i.e., 38.4 seconds), the figures show that it is unable to detect any flooding attack. Since the flooding attack traffic constantly occupies a fixed portion of the bottleneck link capacity, the incoming TCP data traffic adapts to the remaining bandwidth without significant fluctuations.

5.5.2. The DTW Method. Besides filtering noise in the incoming traffic, the DTW method also modifies the original dynamic time warping algorithm by introducing an adaptive penalty $p$ to avoid matching patterns with different periods [25]. We realized the DTW method based on the implementation of the original dynamic time warping algorithm [26]. For the experiment setup, we have employed the same parameters suggested in [25, Section 3.6]. In particular, we have set the noise filter threshold $\beta_{2}=0.3$ and the penalty 


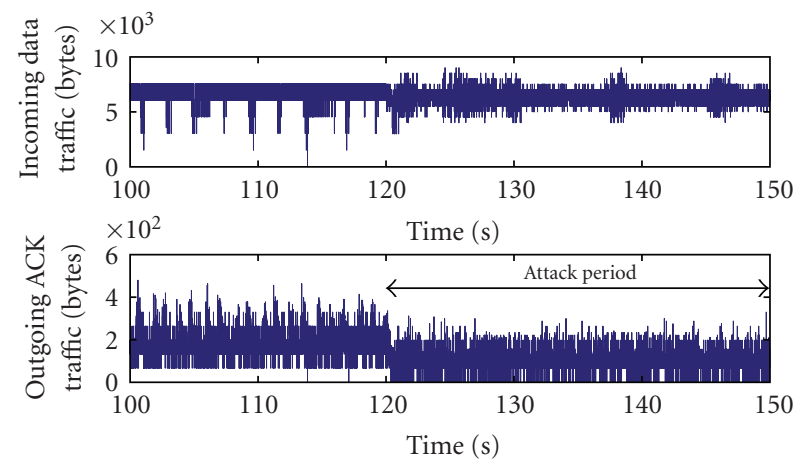

(a) A flooding attack
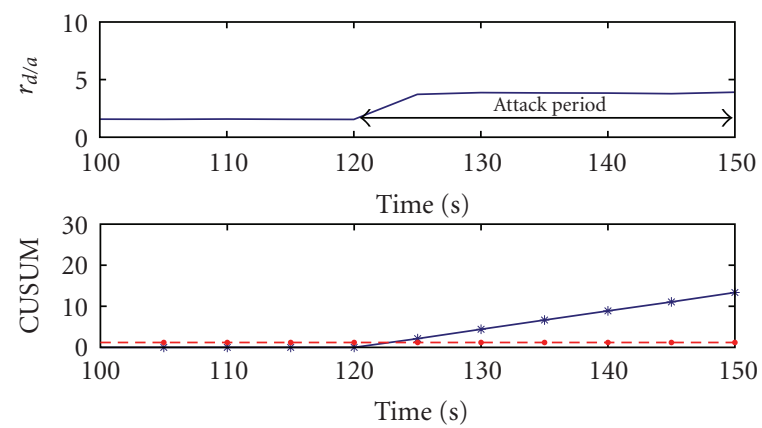

* CUSUM value

- CUSUM threshold $=2.5$

(c) $r_{d / a}$
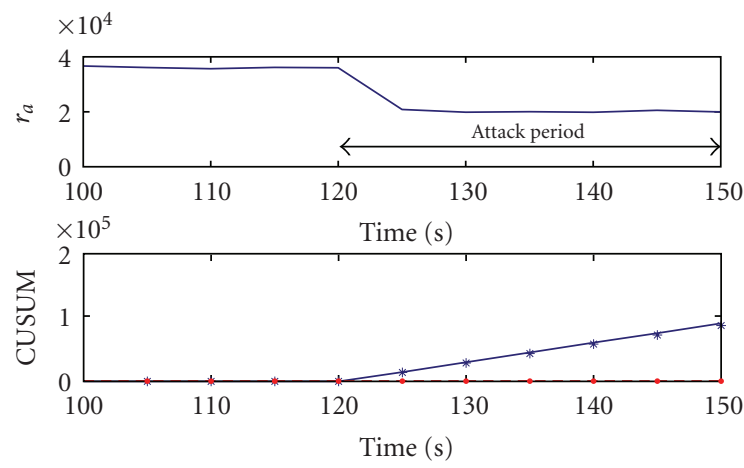

* CUSUM value

$\ldots$ CUSUM threshold $=652.4$

(b) $r_{a}$
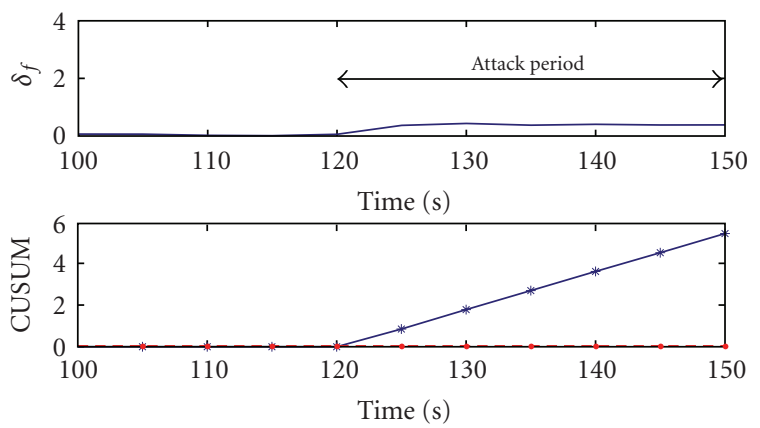

* CUSUM value

$\ldots$ CUSUM threshold $=0.0391$

(d) $\delta_{f}$

Figure 6: Detecting flooding attacks using Vanguard.
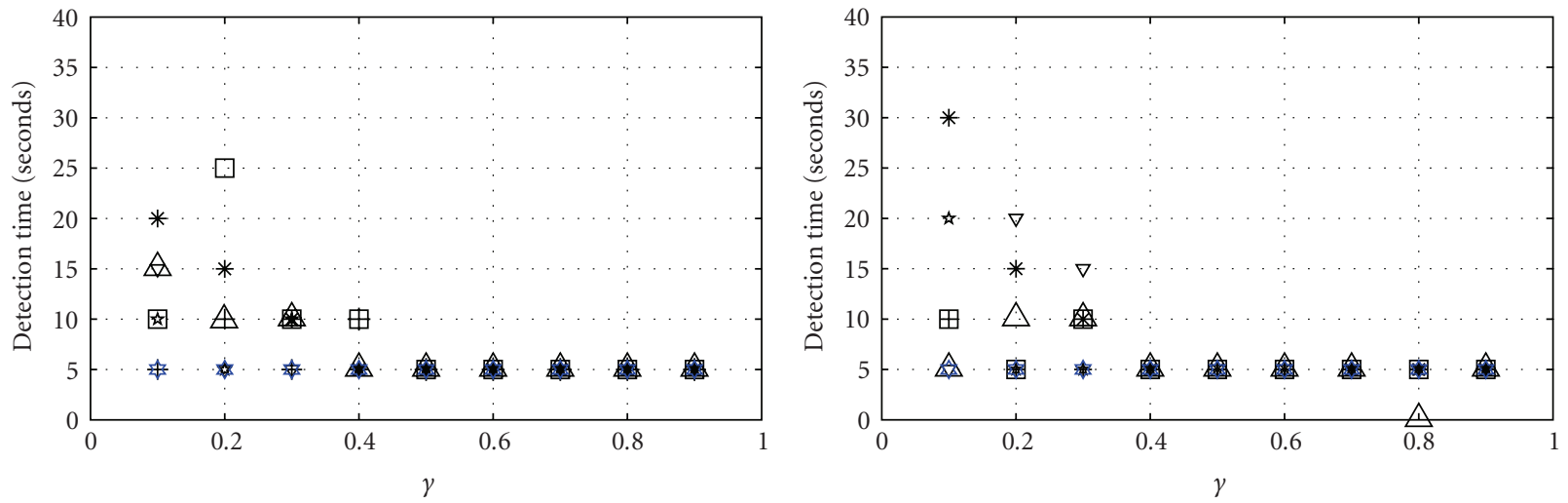

$\square$ Pulsing $\left(T_{\text {on }}=150 \mathrm{~ms}, R_{a}=20 \mathrm{M}\right)$

$\triangle$ Pulsing $\left(T_{\text {on }}=150 \mathrm{~ms}, R_{a}=40 \mathrm{M}\right)$

Pulsing $\left(T_{\text {on }}=150 \mathrm{~ms}, R_{a}=20 \mathrm{M}\right)$

$\triangle$ Pulsing $\left(T_{\text {on }}=150 \mathrm{~ms}, R_{a}=40 \mathrm{M}\right)$

+ Pulsing $\left(T_{\mathrm{on}}=200 \mathrm{~ms}, R_{a}=20 \mathrm{M}\right)$

$+\operatorname{Pulsing}\left(T_{\mathrm{on}}=200 \mathrm{~ms}, R_{a}=20 \mathrm{M}\right)$

* Pulsing $\left(T_{\text {on }}=200 \mathrm{~ms}, R_{a}=40 \mathrm{M}\right)$

* Pulsing $\left(T_{\mathrm{on}}=250 \mathrm{~ms}, R_{a}=20 \mathrm{M}\right)$

$\nabla$ Pulsing $\left(T_{\mathrm{on}}=250 \mathrm{~ms}, R_{a}=40 \mathrm{M}\right)$

is Flooding

* Pulsing $\left(T_{\mathrm{on}}=200 \mathrm{~ms}, R_{a}=40 \mathrm{M}\right)$

\Pulsing $\left(T_{\mathrm{on}}=250 \mathrm{~ms}, R_{a}=20 \mathrm{M}\right)$

$\nabla$ Pulsing $\left(T_{\mathrm{on}}=250 \mathrm{~ms}, R_{a}=40 \mathrm{M}\right)$

is Flooding

(a) Periodic pulsing attacks and flooding attacks

(b) Stochastic pulsing attacks and flooding attacks

FIGURE 7: Average detection time for pulsing and flooding attacks using Vanguard. 


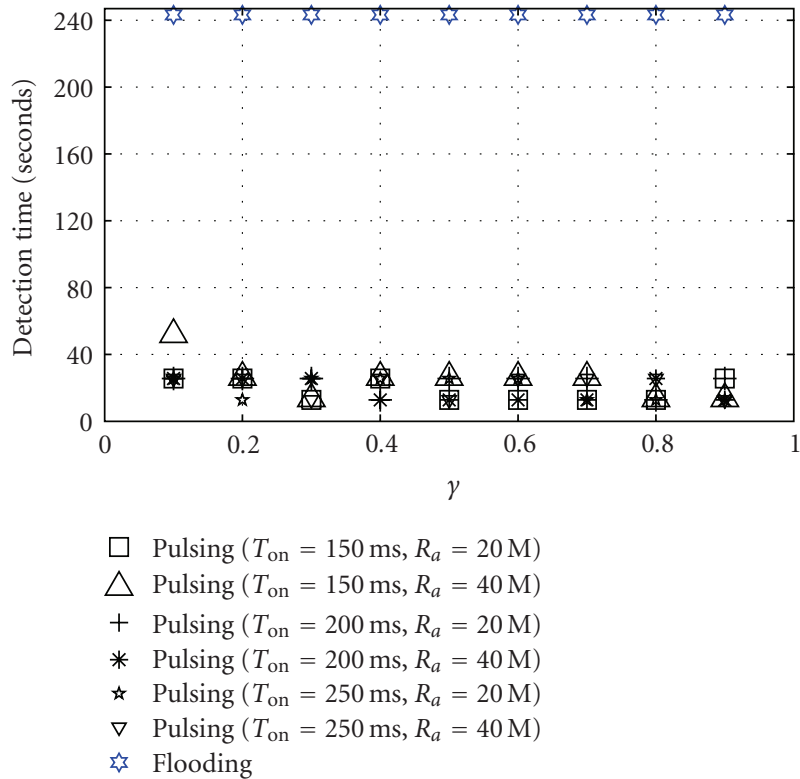

(a) Periodic pulsing attacks and flooding attacks

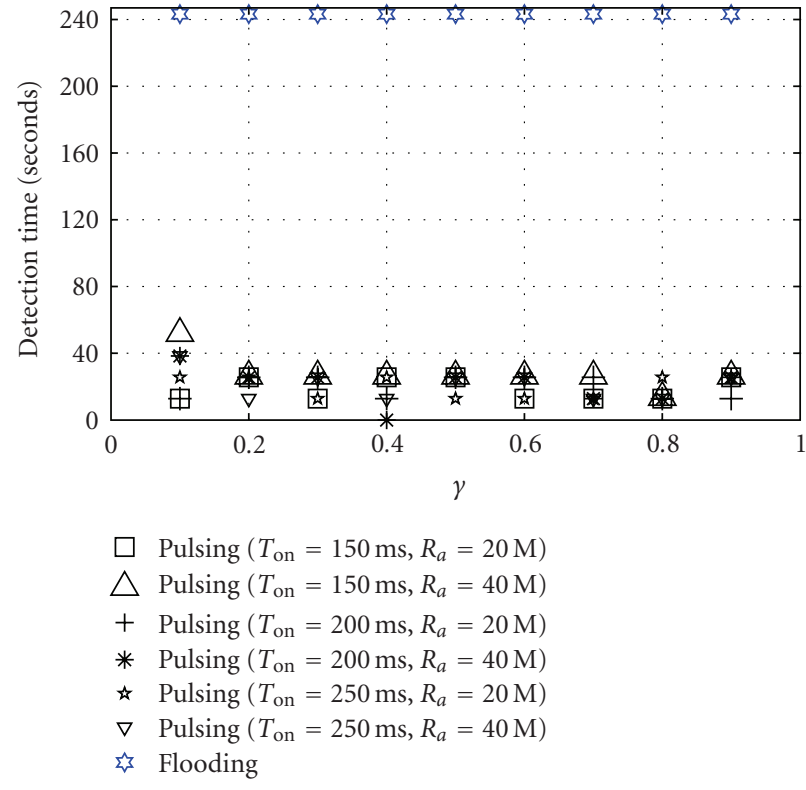

(b) Stochastic pulsing attacks and flooding attacks

FIGURE 8: Average detection time for pulsing and flooding attacks using the WCM method.

value $p=0.01$. The period and the burst width of the lowrate attack signature template are 1.2 seconds and 0.2 second, respectively.

Figure 9 reports the DTW value versus the attack cost for the DTW method. The dashed line with $\mathbf{\nabla}(\square)$ is the DTW threshold of 60 (28.01) for the purpose of differentiating between Gaussian (self-similar) legitimate traffic and attack traffic $[11,25]$. If the DTW value is less than the threshold, the algorithm will confirm the presence of a PMDoS attack. The average detection rates with the DTW thresholds of 60 and 28.01 are $87.18 \%$ and $75.21 \%$, respectively, which are less than what can be achieved by Vanguard and the WCM method. Similar to the WCM method, the DTW method also cannot detect any flooding attack because it was designed specifically for the shrew attack by matching the pattern of the incoming TCP data traffic with the shrew attack traffic.

5.5.3. The STM Method. Figure 10 shows the values of $F(60 \%)$ versus the attack cost for the STM method. In [8], $F(p)$ is defined as the frequency at which the normalized cumulative spectrum captures $p \%$ of the power. $F(p)$ is mainly used for comparing power spectral graphs. In our experiments, we adopt $F(60 \%)$ used in [8]. The experiment results show that the values of $F(60 \%)$ for the pulsing attacks do not concentrate on a small range. Instead, they spread from low frequencies to high frequencies. Therefore, the STM method cannot detect a PMDoS attack based on a static, small range of frequencies as in the case of shrew attacks.

5.5.4. False Positive Rates. We have also evaluated the FPRs for the WCM, DTW, and STM methods using the 62 and 49 TCP flows from the same LBNL and WIDE trace sets, respectively, for the evaluation of Vanguard's FPR. The methods' configuration settings remain unchanged. Table 1 summarizes the results for the three methods. We have also shown Vanguard's FPRs for comparison. Among the four methods, Vanguard achieves the FPRs less than 3\% for both trace sets. The WCM method also achieves low FPRs for the WIDE trace set because it does not contain significant fluctuations of data traffic and abnormal declines in the ACK traffic.

The DTW method, on the other hand, shows the most disappointing performance for both sets of TCP flows with the Gaussian and self-similar thresholds. We note that the thresholds were determined from simulated traffic which may deviate significantly from the realistic traffic. Moreover, our FPR evaluation was based only on the TCP flows for which the data and ACK packets were present, but the DTW method does not have this requirement for the threshold computation. Therefore, we have repeated the evaluation with a DTW threshold $\eta_{44 \%}^{\mathrm{DTW}}$ using the minimum DTW values of the $44 \%$ of the TCP flows for each trace set. By using $\eta_{44 \%}^{\mathrm{DTW}}$ of 5.355 (5.530) for the LBNL (WIDE) trace set, the FPR for the remaining 35 (27) TCP flows drops to $8.57 \%$ $(0 \%)$.

5.5.5. Time Complexity Analysis. Having a low computational complexity is a very important consideration in designing a practical detection system. Therefore, we compare the time complexity for Vanguard and other methods in this section. Table 2 summarizes the comparison results, where $N$ is the number of observations collected in a detection window. Both Vanguard and the WCM methods achieve the lowest time complexity. Before considering each method, we first note that the lowest time complexity for 


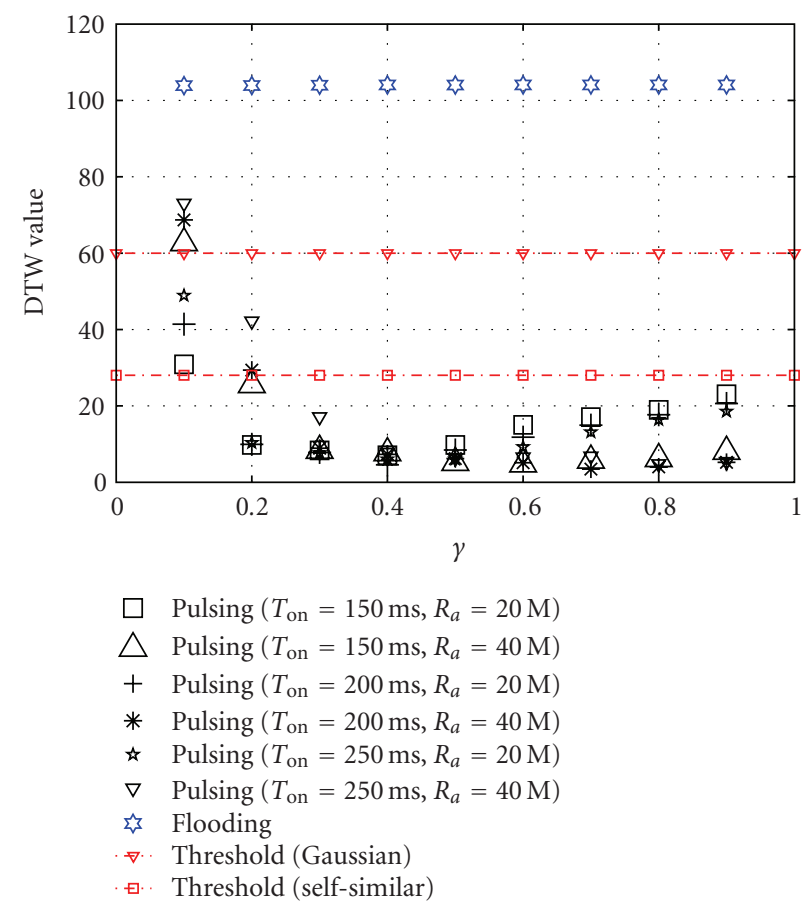

(a) Periodic pulsing attacks and flooding attacks

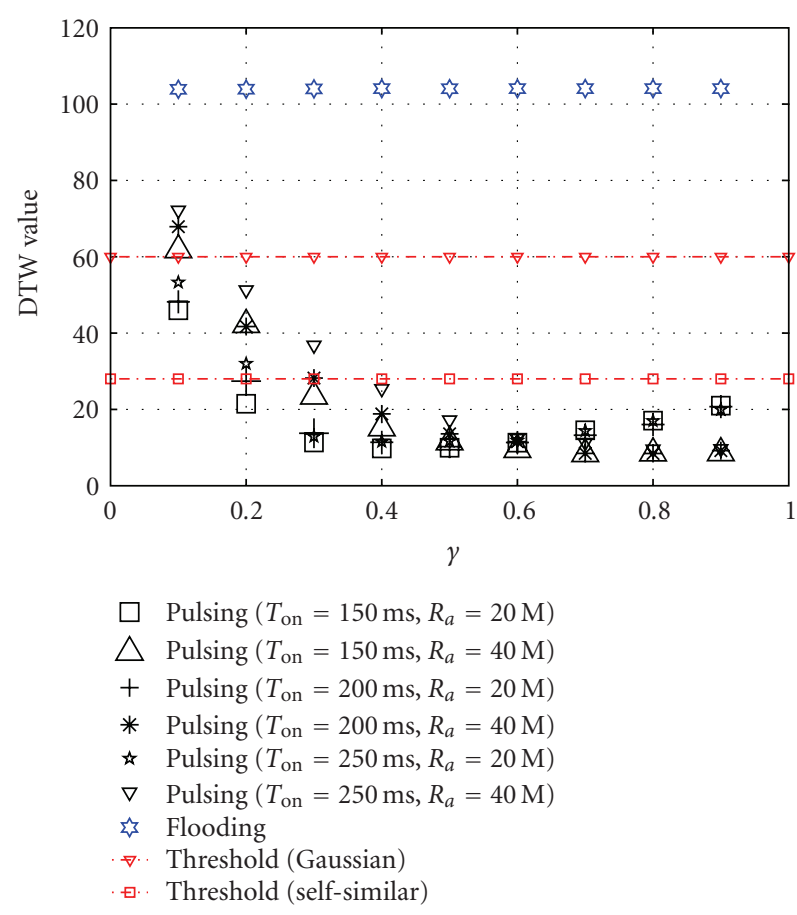

(b) Stochastic pulsing attacks and flooding attacks

FIGURE 9: Average detection time for pulsing and flooding attacks using the DTW method.

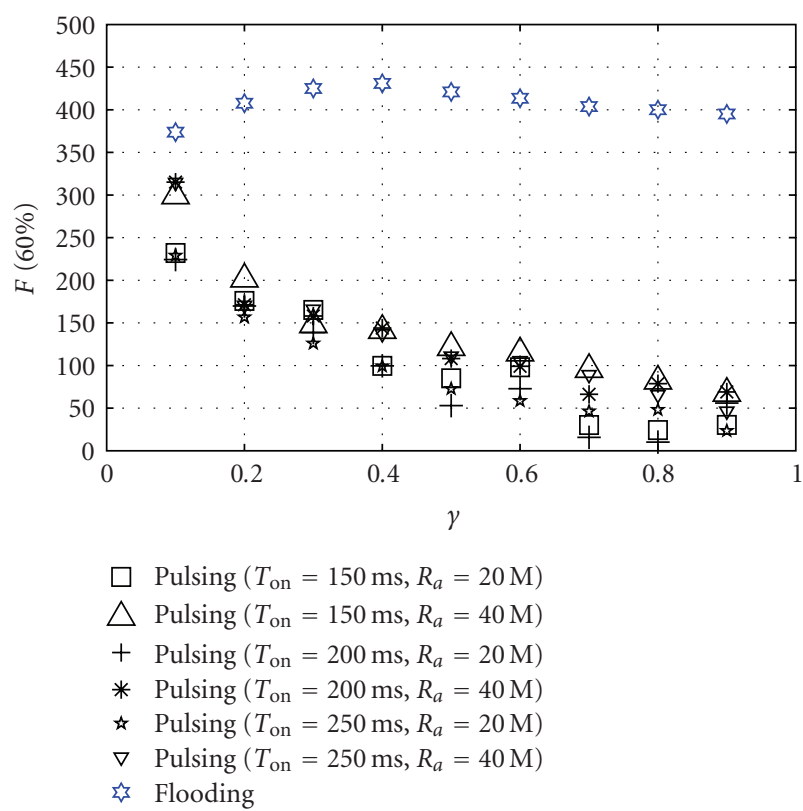

(a) Periodic pulsing attacks and flooding attacks

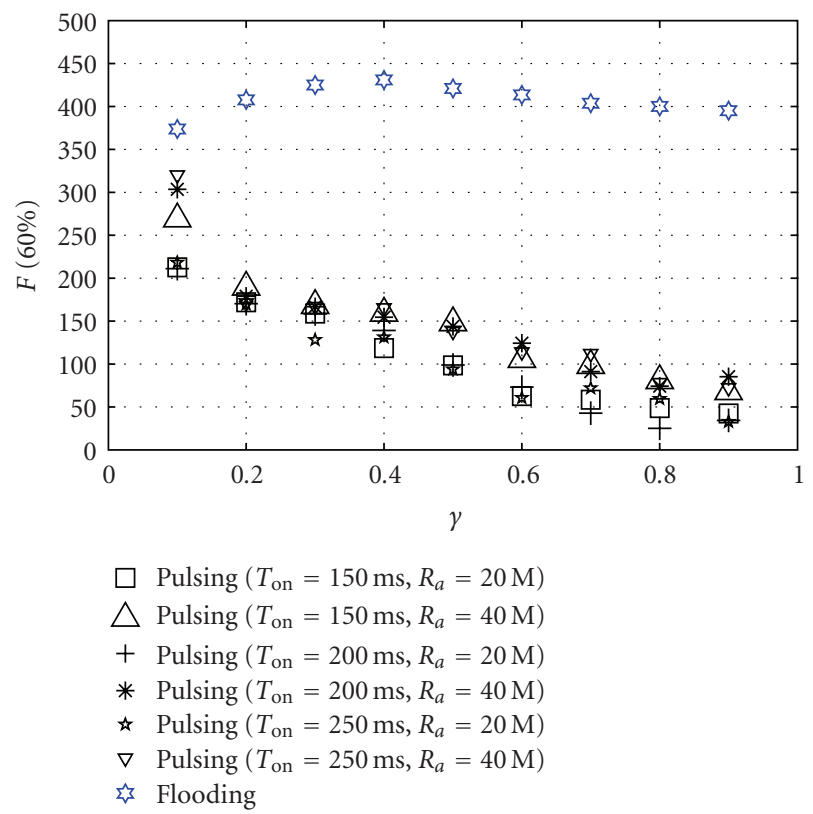

(b) Stochastic pulsing attacks and flooding attacks

FIGURE 10: Average detection times for pulsing and flooding attacks using the STM method.

the methods under consideration is $\Theta(N)$ because their detection decisions are all based on $N$ observations.

Vanguard's time complexity is $\Theta(N)$ which can be obtained according to the following. Recall that there are three statistics to compute. The statistics $r_{a}(n)$ and $r_{d}(n)$ are updated upon receiving each observation. For $\delta_{d}(n)$, by using identical bin size, the respective bin can be quickly located for each observation received. After that, the burden of computing $\delta_{d}(n)$ is determined by $B$, which is usually less than $N$. 
TABLE 1: A comparison of the detection methods' false positive rates.

\begin{tabular}{lcc}
\hline Detection methods & LBNL & WIDE \\
\hline Vanguard & $1.62 \%$ & $2.04 \%$ \\
WCM & $5 \%$ & $2.04 \%$ \\
STM & $16.07 \%$ & $32.39 \%$ \\
DTW (Gaussian) & $93.55 \%$ & $100 \%$ \\
DTW (self-similar) & $89.66 \%$ & $100 \%$ \\
DTW $\left(\eta_{44 \%}^{\text {DTW }}\right)$ & $8.57 \%$ & $0 \%$ \\
\hline
\end{tabular}

TABle 2: A comparison of the detection methods' time complexity.

\begin{tabular}{lc}
\hline Detection methods & Time complexity \\
\hline Vanguard & $\Theta(N)$ \\
WCM & $\Theta(N)$ \\
STM & $\Theta(N \log N)$ \\
DTW & $\Theta\left(N^{2}\right)$ \\
\hline
\end{tabular}

The WCM method's time complexity is given by that of the discrete wavelet transform which is $\Theta(N)$ [27]. The STM method's time complexity is determined mainly by the amount of work on computing the power spectrum density, which is $\Theta(N \log N)$ [28]. For the DTW method, the computational complexity of the autocorrelation processing is $\Theta\left(N^{2}\right)$ and that of DTW is $\Theta(N L)$, where $L$ is the length of selected signatures for the shrew attack. The method's time complexity is therefore $\Theta\left(N^{2}\right)$.

\section{Conclusions and Future Work}

In this paper we have proposed Vanguard to detect a class of pulsing and flooding DoS attacks, which we refer to them collectively as polymorphic DoS (PMDoS) attacks. Unlike the traditional flooding-based DoS attack, the PMDoS attack may exhibit various traffic patterns that cannot be easily detected. The detection engine in Vanguard is based on three traffic anomalies. A single anomaly is used for the case of observable attack traffic, whereas the other two cases are used for the case of unobservable attack traffic. We have subsequently designed a detection algorithm based on the relevant statistics and a CUSUM algorithm to detect abrupt changes in them.

To evaluate Vanguard on a testbed, we have implemented it as a Snort plug-in. The experiment results have confirmed Vanguard's capability of detecting a wide range of PMDoS attacks with a short detection time. We have also evaluated other detection methods on the testbed. None of them could effectively detect the diverse attack scenarios as Vanguard does because they were designed for either specific DoS attacks or restricted attack scenarios. In the future work, we will mainly concentrate on applying effective machine learning algorithms to improve the detection performance.

\section{Acknowledgments}

The work described in this paper was partially supported by a grant from the Research Grant Council of the Hong Kong
Special Administrative Region (Project no. PolyU 5080/02E), a grant from the Areas of Excellence Scheme established under the University Grants Committee of the Hong Kong Special Administrative Region (Project no. AoE/E-01/99), a grant from the Cisco University Research Program Fund at Community Foundation Silicon Valley, and a PolyU Research Grant (Project no. G-T848). The authors are also indebted to the three reviewers and Professor Chin-Tser Huang for meticulously reviewing the manuscripts and for offering many useful comments and questions to improve the readability and technical accuracy of this paper.

\section{References}

[1] A. Kuzmanovic and E. W. Knightly, "Low-rate TCP-targeted denial of service attacks: the shrew vs. the mice and elephants," in Proceedings of the Conference on Applications, Technologies, Architectures, and Protocols for Computer Communications (SIGCOMM '03), pp. 75-86, Karlsruhe, Germany, August 2003.

[2] M. Guirguis, A. Bestavros, and I. Matta, "Exploiting the transients of adaptation for RoQ attacks on internet resources," in Proceedings of the 12th IEEE International Conference on Network Protocols (ICNP '04), pp. 184-195, Berlin, Germany, October 2004.

[3] X. Luo and R. K. C. Chang, "On a new class of pulsing denial-of-service attacks and the defense," in Proceedings of the Network and Distributed System Security Symposium (NDSS '05), pp. 1-19, San Diego, Calif, USA, February 2005.

[4] A. Shevtekar, K. Anantharam, and N. Ansari, "Low rate TCP denial-of-service attack detection at edge routers," IEEE Communications Letters, vol. 9, no. 4, pp. 363-365, 2005.

[5] Y.-K. Kwok, R. Tripathi, Y. Chen, and K. Hwang, "HAWK: halting anomalies with weighted choKing to rescue wellbehaved TCP sessions from shrew DDoS attacks," in Proceedings of the 3rd International Conference on Computer Network and Mobile Computing (ICCNMC '05), pp. 423-432, Zhangjiajie, China, August 2005.

[6] Y. Xu and R. Guérin, "On the robustness of router-based denial-of-service (DoS) defense systems," ACM SIGCOMM Computer Communication Review, vol. 35, no. 3, pp. 47-60, 2005.

[7] The open source network intrusion detection system: Snort, http://www.snort.org.

[8] A. Hussain, J. Heidemann, and C. Papadopoulos, "A framework for classifying denial of service attacks," in Proceedings of the Conference on Applications, Technologies, Architectures, and Protocols for Computer Communications (SIGCOMM '03), pp. 99-110, Karlsruhe, Germany, August 2003.

[9] Y. Chen, K. Hwang, and Y.-K. Kwok, "Filtering of shrew DDoS attacks in frequency domain," in Proceedings of the 30th Anniversary of IEEE Conference on Local Computer Networks (LCN '05), pp. 786-793, Sydney, Australia, November 2005.

[10] Y. Chen and K. Hwang, "Collaborative detection and filtering of shrew DDoS attacks using spectral analysis," Journal of Parallel and Distributed Computing, vol. 66, no. 9, pp. 11371151, 2006.

[11] H. Sun, J. C. S. Lu, and D. K. Y. Yau, "Defending against low-rate TCP attacks: dynamic detection and protection," in Proceedings of the 12th IEEE International Conference on Network Protocols (ICNP '04), pp. 196-205, Berlin, Germany, October 2004. 
[12] J. Mirkovic, G. Prier, and P. Reiher, "Attacking DDoS at the source," in Proceedings of the 10th IEEE International Conference on Network Protocols (ICNP '02), pp. 312-321, Paris, France, November 2002.

[13] K. Chandrayana and S. Kalyanaraman, "Uncooperative congestion control," in Proceedings of the ACM Joint International Conference on Measurement and Modeling of Computer Systems (SIGMETRICS '04), pp. 258-269, New York, NY, USA, June 2004.

[14] M. Allman, V. Paxson, and W. Stevens, "TCP congestion control," Tech. Rep. RFC 2581, IETF, San Francisco, Calif, USA, April 1999.

[15] M. A. Stricker and M. Orengo, "Similarity of color images," in Storage and Retrieval for Image and Video Databases III, vol. 2420 of Proceedings of SPIE, pp. 381-392, San Jose, Calif, USA, February 1995.

[16] A. W. M. Smeulders, M. Worring, S. Santini, A. Gupta, and R. Jain, "Content-based image retrieval at the end of the early years," IEEE Transactions on Pattern Analysis and Machine Intelligence, vol. 22, no. 12, pp. 1349-1380, 2000.

[17] B. Brodsky and B. Darkhovsky, Non-Parametric Statistical Diagnosis: Problems and Methods, Kluwer Academic Publishers, Dordrecht, The Netherlands, 2000.

[18] L. Rizzo, "Dummynet: a simple approach to the evaluation of network protocols," ACM SIGCOMM Computer Communication Review, vol. 27, no. 1, pp. 31-41, 1997.

[19] S. Floyd and V. Jacobson, "Random early detection gateways for congestion avoidance," IEEE/ACM Transactions on Networking, vol. 1, no. 4, pp. 397-413, 1993.

[20] S. Floyd, "RED: Discussions of Setting Parameters," 1997, http://www.icir.org/floyd/REDparameters.txt.

[21] "WinPcap: The Windows Packet Capture Library," 2008, http://www.winpcap.org.

[22] "NLANR/DAST: Iperf 1.7.0 - The TCP/UDP bandwidth measurement tool," 2003, http://dast.nlanr.net/Projects/Iperf.

[23] "Lawrence Berkeley National Laboratory (LBNL) and ICSI,"LBNL's internal enterprise traffic, 2005, http://www.icir.org/enterprise-tracing.

[24] MAWI Working Group, "Packet traces from WIDE backbone," 2006, http://tracer.csl.sony.co.jp/mawi.

[25] H. Sun, J. C. S. Lui, and D. K. Y. Yau, "Distributed mechanism in detecting and defending against the low-rate TCP attack," Computer Networks, vol. 50, no. 13, pp. 2312-2330, 2006.

[26] J.-S. R. Jang and H.-R. Lee, "A general framework of progressive filtering and its application to query by singing/humming," IEEE Transactions on Audio, Speech, and Language Processing, vol. 16, no. 2, pp. 350-358, 2008.

[27] M. Weeks and M. Bayoumi, "Discrete wavelet transform: architectures, design and performance issues," The Journal of VLSI Signal Processing, vol. 35, no. 2, pp. 155-178, 2003.

[28] A. Oppenheim, A. Willsky, and S. Nawab, Signals and Systems, Prentice-Hall, Englewood Cliffs, NJ, USA, 2nd edition, 1996. 